\title{
Model-based therapeutics for the cardiovascular system - a clinical focus
}

\author{
Christopher. E. Hann*, J. Geoffrey Chase*, Thomas Desaive**, Christina Starfinger*, Bernard Lambermont**, \\ Alexandre Ghuysen**, Philippe Kolh** and Geoffrey M. Shaw ***
}

\author{
*Mechanical Eng, Centre for Bio-Engineering, University of Canterbury, Christchurch, New Zealand \\ (Tel: +64-3-364-7001; e-mail: Chris.Hann@canterbury.ac.nz). \\ **Hemodynamics Research Laboratory, University of Liege, Belgium (e-mail: tdesaive@ulg.ac.be) \\ ***Department of Intensive Care, Christchurch Hospital, Christchurch, New Zealand
}

\begin{abstract}
This paper focuses on clinical results of a model-based cardiac diagnosis and therapy methodology. The method allows for accurate patient specific identification and prediction of a broad range of hemodynamics typical in the critically ill. Extensive porcine experiments have been performed involving drug induced pulmonary embolism and septic shock including hemofiltration therapy. The model-based methods consistently capture all measured cardiac variables, within 5-10\% error throughout the experiments. Importantly, all the physiologically expected responses to pulmonary embolism and septic shock are captured and compare well to more directly measured but highly invasive procedures. The cardiovascular system model only requires clinical data that is readily available in the Intensive Care Unit. Therefore, the results demonstrate the potential for longer term developing a cardiac diagnosis and therapeutic guidance system for use in critical care at the bedside.
\end{abstract}

\section{INTRODUCTION}

Inadequate diagnosis of cardiac disease and dysfunction is prevalent in critical care and is a significant cause of increased length of stay and death [Angus 2001]. However, detection, diagnosis and treatment is very difficult, with clinicians confronted by a wealth of confusing, contradictory numerical data. The overall goal in this research is to put this clinical data into a readily understood physiological context for clinicians, by using computational models to unmask hidden dynamics and interactions and thus make diagnosis simpler and more robust.

In critical care, cardiac assessment commonly involves the analysis of changes in aortic pressure, cardiac output, ECG and gas exchange measurements relative to a normal or "average" patient. However complex interactions in these measurements can hide the underlying disease state, preventing proper treatment or detection [Dickstein 2005]. Diagnosis is thus often based on potentially incomplete/flawed conceptual models and understanding of attending clinical staff. Hence, the primary problem is that the wealth of available data is not fully utilised to reveal these hidden dynamics and interactions.

This research shifts the focus to the fundamental dynamics of the cardiovascular system (CVS) using computer models and patient specific parameter identification to create patient specific models [Starfinger 2007, 2008a]. The goal is to use the parameters in these models to directly represent the physiological status of the patient. From this computer aided physiological picture, clinical diagnosis is much easier as the data now fits clinical expectations and hidden physiological dynamics and interactions are exposed - a transformation from numerical data to physiology. This approach can thus provide a stronger foundation for treatment selection.

The results of applying the methods to pulmonary embolism and septic shock data with and without hemofiltration are presented and discussed. Hence the cardiac model and system identification approach is shown to be applicable in a wide range of scenarios, proving its potential for longer term implementation in the Intensive Care Unit (ICU) at the bedside.

\section{METHODS}

\subsection{Model}

The CVS model is a lumped parameter, eight chamber model [Starfinger 2008a] and is summarized in Figure 1.

The main focus for this paper is on the clinical results, therefore only a brief description of the model is given here. The model is extended from earlier work [Starfinger 2007] to include extra compartments for the lung capillaries and body capillaries. These additions, separate the venous and arterial systems and resistances to gain a more physiologically accurate description of observed dynamics in the literature. For example two resistances on the arterial and venous sides as seen in Figure 1, are necessary to capture the complex behaviors observed in mechanical or spontaneous breathing [Starfinger 2008b].

\subsection{Parameter identification method}

The goal is to identify the CVS model parameters based only on input data that is available in an ICU. The measured signals used are the pressures in the aorta and pulmonary 
artery $\left(P_{a o}, P_{p a}\right)$, and the volumes in the ventricles $\left(V_{l v}, V_{r v}\right)$. The left and right ventricle pressures are available but are not used. The method is to set up a sequence of linear least squared optimizations based on an integral formulation of the measured or estimated data. The identification process has been previously described [Hann 2006, Starfinger 2006, Starfinger 2007], thus only a summary of the method is given:

- Inputs: Pressures $\left(P_{a o, \max }, P_{a o, \min }, P_{a o, \min }, P_{a o, \min }\right)$ and Volumes $\left(V_{l v, \text { max }}, V_{l v, \text { min }}, V_{l v, \text { max }}, V_{l v, \text { max }}\right)$

- Volume and pressure waveforms are generated by scaling a set of pre-calculated model outputs to best match the maximum/minimum values above.

- $\quad$ The integral-based parameter identification method [Hann 2006] is applied to the scaled signals, and the resulting identified parameters are used to resimulate the model

- The result is a much closer match to the clinical data than the initial pre-calculated model outputs.

The re-simulated waveforms are scaled again and the process is continued until the relative error between the model output and measured data is less than a set tolerance or doesn't improve.

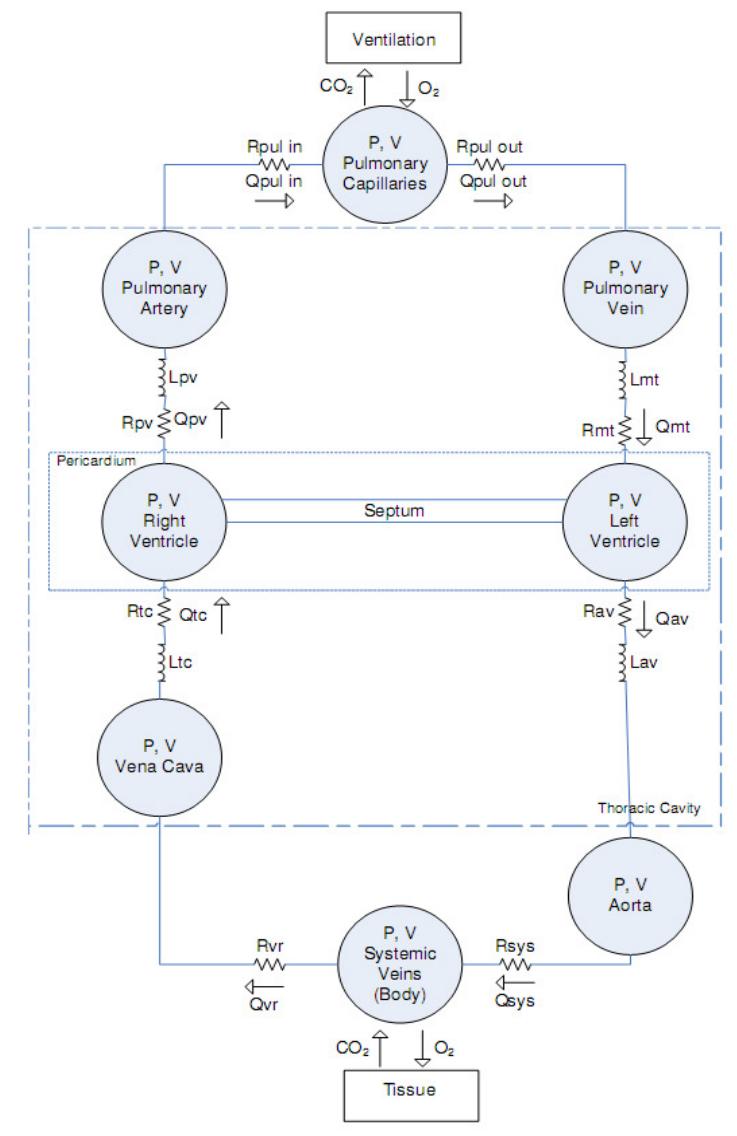

Fig. 1: Overview of the 8 chamber CVS model

\subsection{Pulmonary embolism experiment}

Pulmonary Embolism (PE) usually develops from thrombi that form in the deep veins of the legs, pelvis or arms and dislodge and embolize to the pulmonary arteries. PE is a serious condition that can cause permanent damage to the lung, low oxygen levels and damage to other organs as caused by the decrease in oxygen supply. If a clot is large, or if there are many clots, pulmonary embolism can cause death.

Diagnosing Pulmonary Embolism accurately and in a timely manner is challenging and current diagnostic tests are often very costly and consume a considerable amount of resources. The goal of this study is to demonstrate the applicability of the CVS model for identifying the varying PE disease states from the healthy state to the onset of the disease and fully diseased state.

Under the control of the Ethics committee of the Medical Faculty of the University of Liege, pulmonary embolization was induced in pigs with autologous blood clots [Ghuysen 2007]. The clots were injected every two hours with decreasing concentrations. Aortic pressure and pulmonary artery pressure are measured using micromanometer-tipped catheters while right and left ventricle pressures are measured using 7F, 12 electrodes conductance micro-manometer tipped catheters. This research uses data from 6 pigs in that study. The measurements of the pigs are taken during open chest surgery, and Figure 2 gives an example of the procedure.

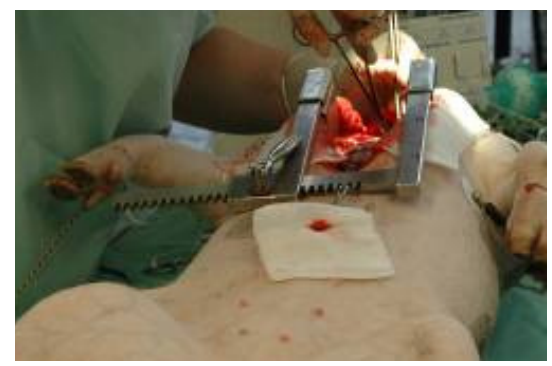

Fig. 2: Open chest surgery on a pig.

\subsection{Septic shock experiments}

Septic shock is characterized by a decreased venous return to the right heart caused by the combined effects of capillary leaks, less stressed systemic volume and loss of circulatory tone leading to systemic arterial resistance. Moreover, venous capacitance and pulmonary resistance are usually increased, which together with a reduced ventricle contractility lead to impaired stroke volume and cardiac output. A decreased systemic arterial resistance therefore leads to a low blood pressure and consequently hypotension. Fluid resuscitation can compensate for the capillary leak and result in increased venous return. Most patients in septic shock after fluid resuscitation thus have a high cardiac output, but a low systemic vascular resistance state [Dellinger 2003], indicating the key diagnostic characteristics and treatments.

Two experiments were done involving 12 healthy pure pietran pigs of either sex weighing from 20 to $30 \mathrm{~kg}$. All the 
animals received a $0.5-\mathrm{mg} / \mathrm{kg}$ endotoxin infusion over 30 minutes (from T0 to T30). Six of the animals received hemofiltration therapy after 60 minutes, and the remaining six received no further intervention. The data taken for each of the experiments is summarized in Equations (1) and (2). For more details on the experimental set up see [Lambermont 2006].

\section{No hemofiltration therapy}

Measured data $\equiv P_{a o}, P_{p a}, P_{l v}, P_{r v}, V_{l v}, V_{r v}$

\section{Hemofiltration therapy}

Measured data $\equiv P_{a o}, P_{p a}, P_{r v}, V_{r v}$

In the case of the data set of Equation (2), the left ventricle volume was assumed to be the same as the right for purposes of identification.

\section{RESULTS AND DISCUSSION}

The identified parameters are used to rerun the CVS model and produce pressure and volume curves, which are then compared to the clinical data. This process is repeated in all the measured periods for both the pulmonary embolism and septic shock experiments using the pig's steady state response data.

\subsection{Pulmonary embolism}

To demonstrate the effectiveness of the model and method the results of one of the pigs are shown and discussed in detail.

\subsubsection{Pig 1: Beginning of experiment - 30 mins}

30 minutes into the pulmonary embolism experiment, the simulation data matches the measured porcine data very well with errors within $4.2 \mathrm{mmHg}$ and $4.4 \mathrm{ml}$ for maximum and minimum pressures and volumes respectively. Fig 4 shows the resulting Pressure-Volume relationships (P-V loops) for the left and right ventricles. Errors in all cases are in the range of $0.15 \%$ to $4.76 \%$.

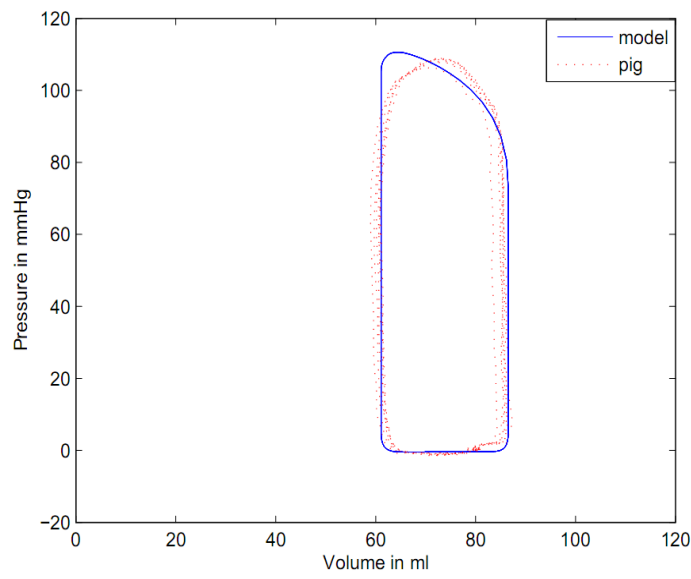

(a) 30 mins left ventricle

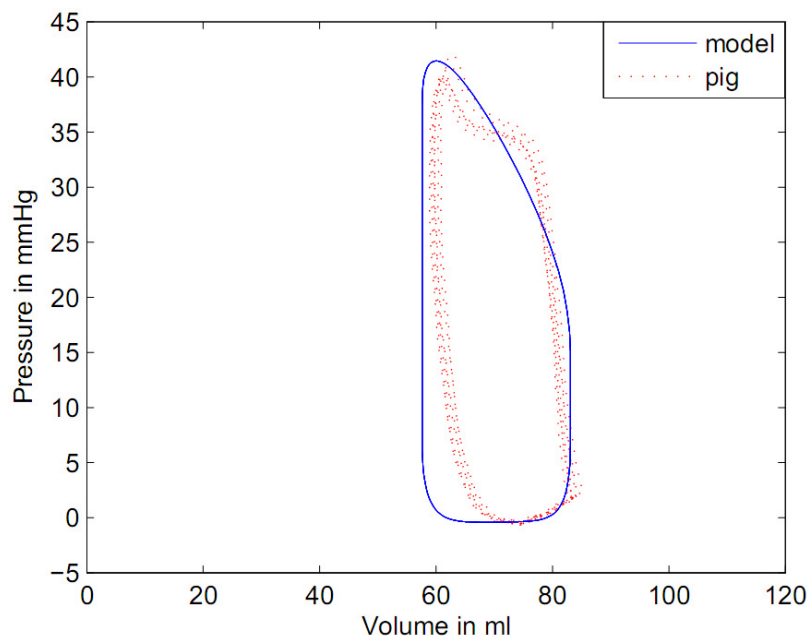

(b) 30 mins right ventricle

Fig. 4: Pig 1, Pressure-Volume Relationship (Dashed is clinical data and solid is identified model)

\subsubsection{Pig 1: During the experiment - 120 minutes}

The P-V loops for the left and right ventricle after 120 were accurately captured with the errors in the maximum pressures and volumes that are typically used to define trends in different disease states within $0.17 \%$ to $4.95 \%$, respectively.

\subsubsection{Pig 1: End of experiment and summary - 180 minutes}

Similarly accurate matches to the P-V loops (not shown) are also obtained at 180 minutes, which was the end of the experiment. Errors in the maximum pressures and volumes are all within $0.20 \%$ and $6.59 \%$ respectively.

The identified subject (pig) specific parameters systemic and pulmonary vascular resistance, Rsys and Rpul, differ significantly between healthy and disease state as Fig 6 shows with Rpul increasing by $261.44 \%$.
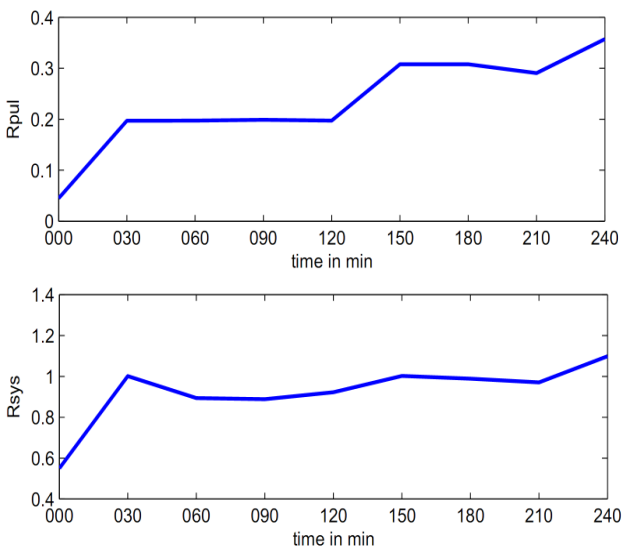

Fig. 6: Pig 1: Pulmonary vascular $\left(R_{p u l}\right)$ and systemic $\left(R_{s y s}\right)$ resistance during pulmonary embolism experiment 


\subsubsection{Summary of results in all pigs}

The results of Figures 3-6 are similar for all the pigs, with a range of pulmonary vascular resistance increase from $89.98 \%$ to $261.44 \%$. Thus, it matches common physiological signs seen in pulmonary embolism as pulmonary arterial obstruction and the release of vasoactive substances increase pulmonary vascular resistance.

Fig 6 shows the ability of the model to pick up reflex response, where the pig increases systemic resistance to help restore blood pressure. The left and right ventricle contractilities $\left(E_{\text {eslvf }}, E_{\text {esrvf }}\right)$ also increased during the pulmonary embolization experiment (not shown). These contractilities are known to be part of reflex response and could be used as an index of ventricular inotropic state.

A final result is the analysis of right ventricular vascular coupling, which is the ratio of contractility $\left(E_{e s, l v f}\right)$ to afterload $\left(R_{s y s}\right)$. Fig 7 shows the mean coupling over all pigs with error bars referring to \pm standard deviation. The model-based measure starts at $2.01 \pm 0.47$ and decrease to $0.70 \pm 0.20$ at the end. These results can be compared to an invasive measurement based on the rapid vena cava occlusion manuever [Lambermont 2006, Ghuysen 2008]. The measurements calculated invasively, start at $2.78 \pm 0.16$ and decrease to $0.72 \pm 0.24$ at the end. Hence the model accurately captures the magnitude of the overall drop further validating the method.

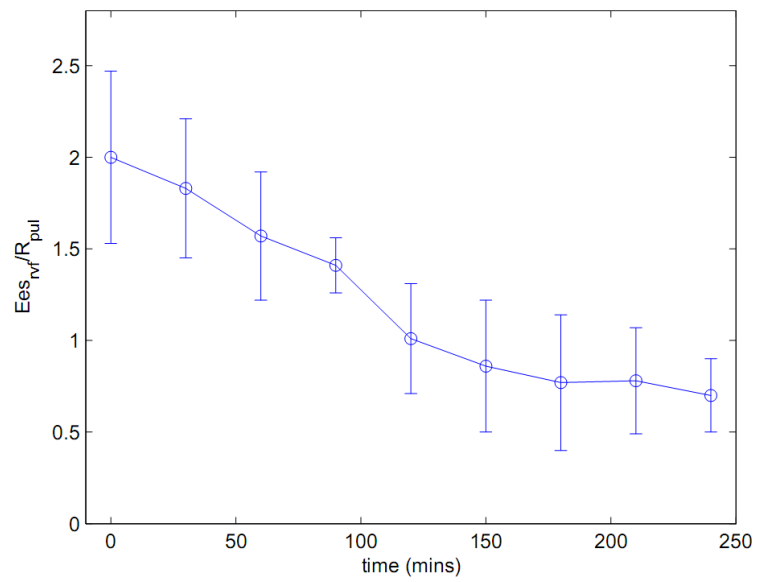

Fig. 7: Model-based right ventricular vascular coupling for all pigs (mean \pm standard deviation)

In the first 90 minutes the pigs preserve coupling reasonably well, but at 120 minutes decoupling occurs, where the contractility can no longer respond to the steadily increasing afterload. The time of 120 minutes also corresponds to the second emboli injection, where in Fig 7 there is a sharp dip. The reason for the preservation of coupling in the initial period could possibly be explained by a dramatic increase in coronary flow.

The measured peak coronary flow before injection is 27.1 $\mathrm{ml} / \mathrm{s}$ which increases to $66.7 \mathrm{ml} / \mathrm{s} 30$ minutes after injection. In other words, to maintain sufficient cardiac output by the coupling mechanism the oxygenation of the right ventricle is increased so that it is less efficient. Eventually the ability of the ventricle to increase contractility in response to increased afterload saturates which causes cardiac output to drop to a level which can no longer support life and the pig dies.

The major known physiological responses due to pulmonary embolism that are captured by the model and identification method are summarized (some results not shown):

- Pulmonary vascular resistance $\left(R_{\text {pul }}\right)$ increases

- Systemic vascular resistance $\left(R_{s y s}\right)$ increases as a reflex response

- Contractilities $\left(E_{\text {eslvf }}, E_{\text {esrvf }}\right)$ increase as a reflex response but in most pigs drop at the end of the experiment where the pig is in a near death state and is no longer able to regulate its circulation

- Right ventricle vascular coupling is preserved 30 minutes after the initial injection as a result of dramatically increased coronary flow

- The model-based coupling measure compares well to the highly invasive measurement using the rapid vena cava occlusion manuever

- Right ventricle expansion index (RVEDV/LVEDV) increases during experiment as the overfilled right ventricle compresses the underfilled left ventricle

- Septum volume $\left(V_{s p t}\right)$ decreases as the septum is shifted towards the left ventricle

- Other model parameters remain largely constant evidencing the ability to distinguish this clinical case without non-physiological parameter values elsewhere in the model

\subsection{Septic shock with and without hemofiltration}

\subsubsection{Without hemofiltration}

The first experiment investigated is with no therapy intervention. In this case there is a reduced dataset, given in Equation (2), since the left ventricle pressure and volume were not measured. Table 1 shows the median absolute percentage errors for the identified minimum and maximum pressure and volume signals (SAP, DAP, SPAP, DPAP, RVESV, RVEDV, RVSV) for the identified re-simulated model over all pigs. Generally, the errors are well below $10 \%$, which is within typical measurement noise levels.

\begin{tabular}{|l|l|l|l|l|l|l|l|}
\hline \multicolumn{1}{|c|}{ Difference in \% for measured and simulated pressures and volumes } \\
\hline & SAP & DAP & SPAP & DPAP & RVEDV & RVESV & RVSV \\
\hline median & 2.87 & 4.33 & 3.02 & 6.40 & 1.37 & 1.55 & 4.71 \\
IQR & 2.89 & 5.82 & 3.99 & 7.98 & 1.43 & 2.09 & 5.11 \\
\hline
\end{tabular}

Table 1: Median absolute percentage error and interquartile range (IQR) in \% for measured and simulated pressures and volumes over all 38 identified segments. SAP/DAP = systolic/diastolic arterial pressure, SPAP/DPAP = systolic/diastolic pulmonary artery pressure, RVEDV = right ventricle end- diastolic volume, RVESV = right ventricle end-systolic volume, RVSV = right ventricle stroke volume. 
Fig 8 shows the pulmonary arterial systolic and diastolic pressure values (SPAP, DPAP) over all times and pigs. The solid lines represent the clinical measurement and the,$+ \square$ markers represent the identified, animal-specific CVS model output for SPAP and DPAP respectively. Similarly, very good matches are obtained, with median absolute percentage errors less than $7 \%$.

Fig 9 shows the mean normalized systemic stressed volume $\left(V_{\text {syseff }}\right)$, and the identified mean normalized systemic vascular $\left(\mathrm{R}_{\text {sys }}\right)$ and pulmonary resistance $\left(R_{\text {pulin }}\right)$, as well as the resistance to venous return $\left(R_{v r}\right)$ overall pigs during the endotoxic shock experiment.

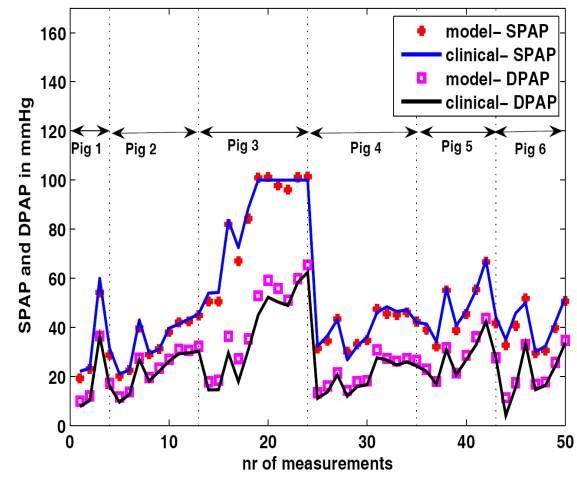

Fig. 8: Clinical (solid lines) vs simulated systolic (cross) and diastolic (box) pulmonary artery pressures (SAP, DAP) over all times and pigs

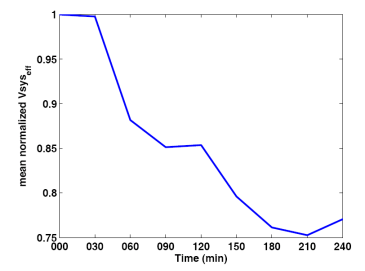

(a) $V_{\text {sys,eff }}$

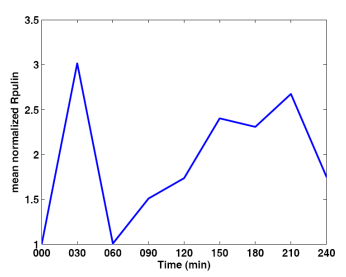

(c) $R_{\text {pulin }}$

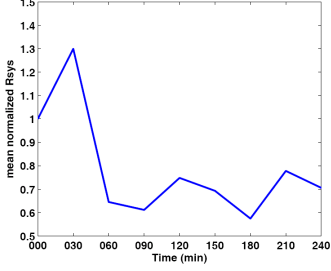

(b) $R_{\text {sys }}$

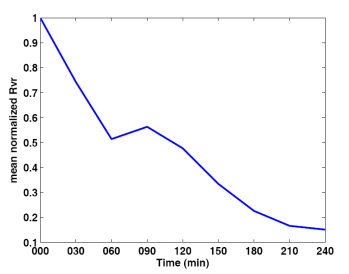

(d) $R_{v r}$
Fig. 9: Mean normalized values for estimated systemic volume Vsys,eff and the identified model parameters systemic vascular resistance Rsys, pulmonary vascular resistance Rpulin and resistance to venous return Rvr over all analyzed pigs during the septic shock experiment.

The results show that, despite the reduced data set and necessary assumptions made, the CVS model is able to accurately identify and re-simulate endotoxic shock. Clinically, and importantly, the identified parameter trends match physiological expectations. For example, Fig 9 shows the model simulated normalized systemic volume and how it decreases during the time course of the experiment, accounting for the loss of circulatory control and hypovolemia seen in septic shock. The data and results obtained from the pig-specific identified models thus match clinical measurements and expectations.

Fig 9 also shows the mean normalized identified trends for the CVS model parameters $R_{\text {sys }}, R_{\text {pulin }}$ and $R_{v r}$ over all analyzed pigs during the experiment. experiment. The overall behaviour, apart from an initial rise, is a significant decrease in $R_{\text {sys }}$ as the septic shock progresses. This trend indicates an initial physiological compensation followed by a failure of circulatory control that is typical of septic shock. From a model-based diagnostics perspective, this result accurately tracks disease onset, initial (successful) compensation and (eventual) physiologic failure, all of which are clinically significant in determining appropriate therapies and dosing.

From 30 to 240 minutes, the pulmonary resistance $R_{\text {pulin }}$ has an increasing trend as the endotoxin infusion takes effect. The initial large increase in the first 30 minutes is due to the short term effect of the sudden onset of septic shock, and the sudden decrease is the initial effect of suddenly stopping the infusion. This response of $R_{\text {pulin }}$ is again consistent with known effects of sepsis and compares with previously published results [Lambermont 2006]. The differences between systemic $\left(\mathrm{R}_{\text {sys }}\right)$, and pulmonary $\left(R_{\text {pulin }}\right)$ behaviours can help guide the balance between vasopressor and fluid resuscitation therapy.

The mean normalized resistance to venous return, $R_{v r}$, decreases consistently during the experiment. This result is expected as the body is trying to restore venous return to improve pressure and increase flow back to the heart. The specific mechanism is through vasodilation, but the model sees this behaviour as a lowered resistance, further validating the methods.

\subsubsection{With hemofiltration and a comparison}

The final experiment investigated is induced septic shock with hemofiltration. A lot of experimental research has shown that hemofiltration can improve hemodynamics and survival in septic shock. The aim is to test the ability of the model to capture the effects of this therapeutic intervention and to validate key parameter trends, including right ventricle vascular coupling.

Fig 10 shows comparisons of the mean normalized right ventricular end-systolic elastance $\left(E_{\text {esrvf }}\right)$ between the endotoxic group (Endo) and hemofiltration group (EndoHF). The top panel is previously reported results [Lambermont 2006] using the rapid vena cava occlusion manoeuvre and the bottom panel is the model identified results. The trends of Endo and EndoHF as well as the differences compare very well between the model-based measure of $E_{\text {esrvf }}$ and [Lambermont 2006]. In particular, the model correctly predicts that for the Endo group the contractility initially rises but after 60 minutes remains relatively low. On the other 
hand the contractility for the EndoHF group increases significantly proving the models ability to predict the impact of the hemofiltration therapy.

Fig 11 gives similar comparisons to that of Fig 10 for the RV-vascular coupling. Overall very good matches are obtained between the model identified parameters and [Lambermont 2006]. Importantly, the model successfully identifies the initial drop in coupling as a result of the endotoxin infusion, and the resulting improvement in the coupling after hemofiltration at 60 minutes. In addition, the model shows a significant diverging of the Endo and EndoHF groups after 90 minutes as is also seen in [Lambermont 2006].

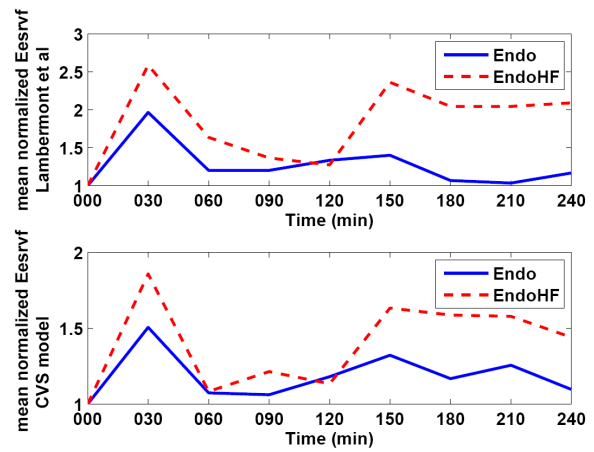

Fig 10: Mean normalized right ventricular end-systolic elastance Eesrvf over all analyzed pigs during the septic shock experiment with (Endo) and without (EndoHF) hemofiltration. Upper panel: results as obtained by (Lambermont et al., 2006), lower panel: results obtained with CVS model and identification process.
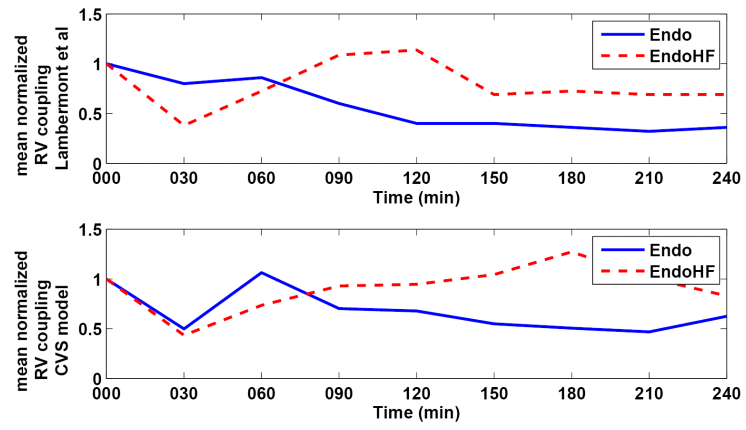

Fig 11: Mean normalized RV-coupling over all analyzed pigs during the septic shock experiment with (Endo) and without (EndoHF) hemofiltration. Upper panel: results as obtained by [Lambermont 2006), lower panel: results obtained with CVS model and identification process.

\section{CONCLUSIONS}

The eight chamber cardiac model accurately captured significant ranges of hemodynamics in clinical animal data. Mean volumes and pressures were identified within $5 \%$ and $8 \%$ respectively with no errors more than $\sim 10 \%$. The model correctly predicted the expected physiological trends in the porcine experiments of pulmonary embolism and septic shock with and without hemofiltration. The model can be identified using a minimal number of common measurements and the identification method is very fast making the approach potentially suitable for real-time diagnostic feedback in a critical care setting. Overall, the results give confidence that a model-based cardiac diagnosis and therapy system is a realistic goal for implementation in the ICU in the future.

\section{REFERENCES}

Angus, D.C., Linde-Zwirble, W.T., Lidicker, J., Clermont, G., Carcillo, J. and Pinsky, M.R. "Epidemiology of severe sepsis in the United States: analysis of incidence, outcome, and associated costs of care," Critical care medicine, Vol. 29, pp. 1303-10, 2001.

Dickstein, K. "Diagnosis and assessment of the heart failure patient: the cornerstone of effective management," European journal of heart failure, Vol. 7, pp. 303-8, 2005.

Starfinger C, Hann, CE, Chase, JG, Desaive T, Ghuysen A and Shaw, G. "Model-based Cardiac Diagnosis of Pulmonary Embolism," Computer Methods and Programs in Biomedicine, Vol 87(1): pp. 46-60, 2007.

Starfinger, C, Chase, JG, Hann, CE, Shaw, GM, Lambermont, B, Ghuysen, A, Kolh, P, Dauby, PC and Desaive, T. "Model-based identification and diagnosis of a porcine model of induced endotoxic shock with hemofiltration," Mathematical Biosciences, Vol 216(2), pp. 132-139, 2008a.

Starfinger, Chase, JG, C, Hann, CE, Shaw, GM, Lambert, P, Smith, BW, Sloth, E, Larsson, A, Andreassen, S and Rees, S. "Model-based identification of PEEP titrations during different volemic levels," Computer Methods and Programs in Biomedicine, Vol 91(2), pp. 135-144, $2008 \mathrm{~b}$.

Hann, CE, Chase, JG and Shaw, GM. "Integral-Based Identification of Patient Specific Parameters for a Minimal Cardiac Model," Computer Methods and Programs in Biomedicine, Vol 81(2), pp. 181-192, 2006.

Ghuysen, A. Lambermont, B. Kolh, P. Tchana-Sato, V., Magis, D. Gerard, P. Mommens, V. Janssen, N. Desaive T. and D'Orio, V. "Alteration of right ventricular-pulmonary vascular coupling in a porcine model of progressive pressure overloading," Shock, Vol 29(2), pp.197-204, 2008.

Dellinger, R.P. "Cardiovascular management of septic shock. Critical Care Medicine, Vol 31(3), pp. 946-955, 2003.

Lambermont, B. Ghuysen, A. Kolh, P. Tchana-Sato, V. Segers, P. Gerard, P. Morimont, P. Magis, D. dogne J.M. Masereel, B. and D'Orio, V. "Effects of endotoxic shock on right ventricular systolic function and mechanical efficiency," Artificial Organs, Vol 30(7), pp. 560-564, 2006. 